\title{
A NEW SOLUTION OF THE GAUSS PROBLEM ON $h\left(s^{2} d\right) / h(d)^{*}$
}

BY GORDON PALL

The following demonstration of the well known formula

$$
h\left(p^{2} d^{\prime}\right)=\sigma^{-1}\left\{p-\left(d^{\prime} \mid p\right)\right\} h\left(d^{\prime}\right)
$$

may be worth noting. Here $h(\Delta)$ denotes the number of classes of primitive integral binary quadratic forms of non-zero discriminant $\Delta ; p$ is any prime $\geqq 2 ; \sigma=1$ if $d^{\prime}<-4$ or $d^{\prime}$ is a square, $\sigma=2$ if $d^{\prime}=-4, \sigma=3$ if $d^{\prime}=-3$; and if $d^{\prime}$ is positive but not square, $\sigma$ is the least positive integer for which $p \mid u_{\sigma},\left(t_{k}, u_{k}\right)$ denoting the successive positive integral solutions of $t^{2}-d^{\prime} u^{2}=4$.

Let $r(n)$ denote the number of sets of representations of $n$ by a representative system of primitive forms of discriminant $d=p^{2} d^{\prime}$. If $q$ is a prime such that $(d \mid q)=1$,

$$
r\left(p^{2} q\right)=2\left\{p-\left(d^{\prime} \mid p\right)\right\} .
$$

For by II (5), (33), (23)-(24), $\dagger$

$$
r\left(p^{2} q\right)=r\left(p^{2}\right) r(q)=2 r\left(p^{2}\right)=2\left\{1+r^{\prime}\left(p^{2}\right)\right\},
$$

where $r^{\prime}\left(p^{2}\right)$ equals the number $p-1-\left(d^{\prime} \mid p\right)$ of solutions $w$ of

$$
(p w)^{2} \equiv p^{2} d^{\prime}\left(\bmod 4 p^{2}\right), \quad \frac{w^{2}-d^{\prime}}{4} \text { prime to } p, \quad\left(0 \leqq p w<2 p^{2}\right) .
$$

By Theorem 4 of I, extended to $d>0$ in II, there is associated with each class (connoted by $K$, say) of primitive forms $f$ of discriminant $p^{2} d^{\prime}$, a unique ambiguous class $C$, or two nonambiguous classes $C$ and $C^{-1}$, of primitive forms $g$ of discriminant $d^{\prime} ; C$ is characterized as representing any prime represented by $K$. By II (13), such forms satisfy, for all integers $n$,

$$
f\left(p^{2} n\right)=\sigma g(n) .
$$

* Presented to the Society, April 6, 1935.

$\dagger$ References are to the writer's two papers: I, Mathematische Zeitschrift, vol. 36 (1933), pp. 321-343; and II, Transactions of this Society, vol. 35 (1933), pp. 491-509. 
Choose $n$ to be a prime $q$ represented by $C$ and prime to $d$. Then $g(q)=2$ if $C$ is ambiguous, $g(q)=1$ if $C \neq C^{-1}$. If a form $f_{1}$ is associated with a form $g_{1}$ not in $C$ or $C^{-1}, f_{1}\left(p^{2} q\right)=\sigma g_{1}(q)=0$. Hence, by (2) and (3), $p^{2} q$ is represented in exactly $\eta\left\{p-\left(d^{\prime} \mid p\right)\right\} \sigma^{-1}$ classes $K$, where $\eta$ is 1 or 2 according as $q$ is represented in only one (ambiguous) or two (reciprocal) primitive classes of discriminant $d^{\prime}$.

MCGill UNIVERSITY

\section{ON A REDUCTION OF A MATRIX BY THE GROUP OF MATRICES COMMUTATIVE WITH A GIVEN MATRIX*}

BY P. L. TRUMP

1. Introduction. Two $n \times n$ matrices $A$ and $B$, with elements in any field $F$, are said to be similar in $F$ if there exists a nonsingular $n \times n$ matrix $S$, with elements in $F$, such that $S^{-1} A S=B$.

Ingraham $\dagger$ has given a method for finding the most general solution, with elements in $F$, of the matrix equation

$$
P(X)=A,
$$

where $P(X)$ is a polynomial with coefficients in $F$, and $A$ is a square matrix with elements in $F$. A certain set of dissimilar solutions $X_{1}, X_{2}, \cdots, X_{k}$ were obtained in terms of which the complete system of solutions was seen to be in the form $S^{-1} X_{i} S$, where $S$ is commutative with $A$. The $X_{i}$ 's are obviously commutative with $A$.

The purpose of this investigation is to determine the conditions under which two $n \times n$ matrices $C$ and $D$ are similar under transformations of the group $[S]$ of non-singular matrices $S$ which are commutative with a certain $n \times n$ matrix $A$, where the matrices $C$ and $D$ are also commutative with $A$. We then seek to describe possible canonical forms to which such matrices

* Presented to the Society, September 4, 1934. This paper with proofs and detail that are omitted here, is on file as a doctor's thesis at the Library of the University of Wisconsin.

$\dagger$ On the rational solutions of the matrix equation $P(X)=A$, Journal of Mathematics and Physics, vol. 13 (1934), pp. 46-50. 